\title{
Robust Tracking of Objects with Dynamic Topology
}

\author{
Padraig Corcoran, Christopher B. Jones \\ School of Computer Science \& Informatics, Cardiff University \\ Wales, UK \\ corcoranp@cardiff.ac.uk
}

\begin{abstract}
In many instances of the object tracking problem the topological properties of objects can change over time. Such changes include the splitting of an object into multiple objects or merging of multiple objects into a single object. We propose a novel tracking model which is robust to such changes. This model is formulated terms of homology theory whereby 0-dimensional homology classes, which correspond to path-connected components, are tracked. A generalisation of this model for tracking spatially close objects lying in an ambient metric space is also proposed. This generalisation is particularly suitable for tracking spatial-temporal phenomena such as weather phenomena. The utility of the proposed model is demonstrated with respect to tracking rain clouds in radar imagery.
\end{abstract}

\section{CCS CONCEPTS}

- Mathematics of computing $\rightarrow$ Algebraic topology; • Information systems $\rightarrow$ Geographic information systems;

\section{KEYWORDS}

Spatial-temporal; Topology; Tracking

\section{ACM Reference Format:}

Padraig Corcoran, Christopher B. Jones. 2018. Robust Tracking of Objects with Dynamic Topology. In 26th ACM SIGSPATIAL International Conference on Advances in Geographic Information Systems (SIGSPATIAL '18), November 6-9, 2018, Seattle, WA, USA. ACM, New York, NY, USA, 4 pages. https: //doi.org/10.1145/3274895.3274922

\section{INTRODUCTION}

Object tracking is necessary in many applications. For example, when making inferences with respect to future weather conditions, it is necessary to track weather phenomena such as a snow storm. Given sensor measurements at a sequence of discrete times, the tracking problem requires the inference of object locations plus correspondences between the same object existing at different discrete times. In many instances of the tracking problem object properties can change over time making it difficult to correctly infer correspondences. For example, when tracking an individual in a video sequence, both the appearance and shape of that individual may change over time. When tracking weather phenomena, the shape and topology of the phenomena may change over time. Changes in the topology include the formation of holes plus the splitting into

Permission to make digital or hard copies of part or all of this work for personal or classroom use is granted without fee provided that copies are not made or distributed for profit or commercial advantage and that copies bear this notice and the full citation on the first page. Copyrights for third-party components of this work must be honored.

For all other uses, contact the owner/author(s).

SIGSPATIAL '18, November 6-9, 2018, Seattle, WA, USA

(C) 2018 Copyright held by the owner/author(s).

ACM ISBN 978-1-4503-5889-7/18/11.

https://doi.org/10.1145/3274895.3274922 and merging of multiple objects. Therefore when tracking objects with changing properties, it is necessary to do so in a manner which is robust to such changes.

In this article we propose a novel tracking model capable of tracking objects whose topological properties change over time. The model assumes objects have been modelled at a sequence of discrete times using a sequence of simplicial complexes. A simplicial complex is a general representation capable of modelling a variety of types of objects such as graphs and point clouds. Consequently, the proposed model is a general model capable of tracking a variety of types of objects. However in this article we focus on the problem of tracking objects corresponding to subsets of $\mathbb{R}^{2}$.

The proposed model formulates the problem of tracking in terms of homology theory whereby it tracks 0-dimensional homology classes which correspond to path-connected components. Persistent homology is a commonly employed approach from homology theory to tracking homology classes of given dimension. However, in its native form, persistent homology is only capable of inferring the appearance and subsequent disappearance of such classes and cannot infer their locations [4]. Generalising persistent homology to overcome this limitation was identified as a challenge in [2] and represents the main contribution of this article.

The layout of this article is as follows. Section 2 describes the proposed tracking model. This section also describes a generalisation of this model for tracking objects in an ambient metric space where objects correspond to sets of spatially close homology classes. For many real world tracking problems, such as tracking weather phenomena, this is the more appropriate model. Section 3 demonstrates application of the model to tracking objects in rainfall radar imagery.

\section{TRACKING MODEL}

This section presents the proposed tracking model. The model assumes that the objects have been modelled at a sequence of discrete times using a sequence of simplicial complexes. In most real world scenarios this sequence will be obtained by performing a triangulation of the objects in question where triangulation is the process of constructing a simplicial complex representation. This section is structured as follows. Section 2.1 presents background material on homology theory and formulates the problem of tracking in terms of computing maps between 0-dimensional homology classes. A fundamental component of computing these maps is the pullback of the zig-zag diagram which is explained in section 2.2. Subsequently, section 2.3 describes how the pullback is employed to compute the maps in question. Section 2.4 describes how object tracking is inferred from these maps. Finally, section 2.5 presents a generalisation of the proposed model for tracking objects in an ambient metric space where objects correspond to sets of spatially close homology classes. 


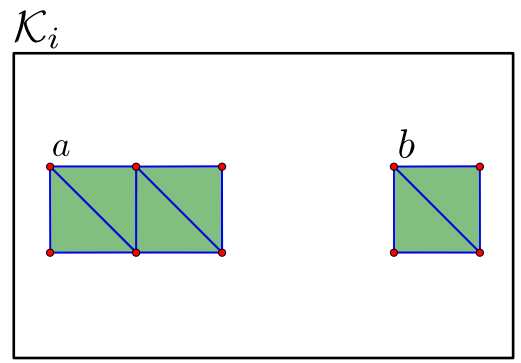

(a)

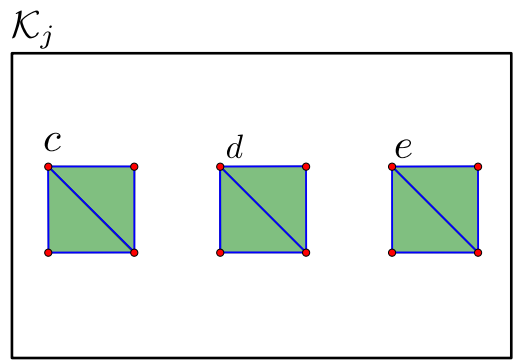

(b)

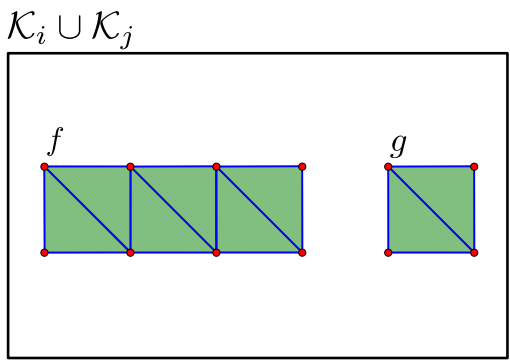

(c)

Figure 1: Two simplicial complexes $\mathcal{K}_{i}$ and $\mathcal{K}_{j}$ and their union $\mathcal{K}_{i} \cup \mathcal{K}_{j}$ are displayed in (a), (b) and (c) respectively. In each figure red dots represent 0 -simplices, blue lines represent 1-simplices and green triangles represent 2-simplices.

\subsection{Model Formulation}

An (abstract) simplicial complex $\mathcal{K}$ is a finite collection of sets such that for each $\sigma \in \mathcal{K}$ all subsets of $\sigma$ are also contained in $\mathcal{K}$. Each element $\sigma \in \mathcal{K}$ is called a simplex or $k$-simplex where $k=|\sigma|-1$ is the dimension of the simplex. The faces of a simplex $\sigma$ correspond to all simplices $\tau$ where $\tau \subset \sigma$. The dimension of a simplicial complex $\mathcal{K}$ is the largest dimension of any simplex $\sigma \in \mathcal{K}$.

A $p$-chain on a simplicial complex $\mathcal{K}$ is defined in Equation 1 where each $\sigma_{i} \in \mathcal{K}$ is a $p$-simplex and each $\lambda_{i}$ is an element in a specified field. The set of $p$-chains forms a group called the chain group $C_{p}(\mathcal{K})$. The boundary map $\partial_{p}$ is a map from a $p$-simplex to the sum of its $(p-1)$-simplex faces as defined in Equation 2. Here $\left[v_{0}, \ldots, \hat{v}_{i}, \ldots, v_{p}\right]$ is the $(p-1)$-simplex obtained by deleting the 0 simplex $v_{i}$ from the $p$-simplex $\left[v_{0}, \ldots, v_{p}\right]$. This map is distributive and extends to the chain groups giving the sequence of chain groups in Equation 3.

$$
\begin{gathered}
c=\sum \lambda_{i} \sigma_{i} \\
\partial_{p} \sigma=\sum_{i=0}^{p}(-1)^{i}\left[v_{0}, \ldots, \hat{v}_{i}, \ldots, v_{p}\right] \\
\ldots \rightarrow C_{p+1}(\mathcal{K}) \stackrel{\partial_{p+1}}{\longrightarrow} C_{p}(\mathcal{K}) \stackrel{\partial_{p}}{\longrightarrow} C_{p-1}(\mathcal{K}) \stackrel{\partial_{p-1}}{\longrightarrow} \ldots
\end{gathered}
$$

A $p$-chain $c$ is a $p$-cycle if $\partial c=0$ and a $p$-boundary if there exists a $(p+1)$-chain $d$ where $c=\partial d$. The sets of all $p$-cycles and $p$-boundaries form groups which are denoted $Z_{p}(\mathcal{K})$ and $B_{p}(\mathcal{K})$ respectively. Each of these groups is a subgroup of $C_{p}(\mathcal{K})$. As a consequence of the fact $\partial_{p+1} \partial_{p}=0$, it can be proved that $B_{p}(\mathcal{K}) \subseteq Z_{p}(\mathcal{K})$. The quotient group $H_{p}(\mathcal{K})=Z_{p}(\mathcal{K}) / B_{p}(\mathcal{K})$ is a vector space and is called the $p$-dimensional homology group of $\mathcal{K}$. The elements of $H_{p}(\mathcal{K})$ are called $p$-dimensional homology classes. Each homology class is an equivalence class over cycles where two cycles in the same homology class are said to be homologous. This means they differ by a boundary. A homology class of $H_{p}(\mathcal{K})$ corresponds to a $p$-dimensional hole in the simplicial complex $\mathcal{K}$. Note that, a homology class of $H_{0}(\mathcal{K})$ corresponds to a path-connected component in the simplicial complex $\mathcal{K}$; this is a consequence of the fact that all 0 -simplices in a path-connected component differ by the boundary of a 1-chain equal to the sum of 1-simplices connecting the 0 -simplices in question. The focus of this article is tracking objects which we define as path-connected components; therefore we consider the 0 -dimensional homology classes and ignore higher order homology classes.

There exist a number of algorithms to compute the number and locations of the homology classes of $H_{p}(\mathcal{K})$ for a given simplicial complex $\mathcal{K}$ and dimension $p$ [4]. In this work we assume a sequence of $n$ simplicial complexes $\mathcal{K}_{1} \ldots \mathcal{K}_{n}$ obtained by triangulating the objects in question at a sequence of $n$ discrete time steps. We wish to track 0-dimensional homology classes existing within this sequence. Toward this goal, for a given $i$ in the range $i \ldots(n-1)$, we define an injective map $T_{i}$ which maps the homology classes of $H_{0}\left(\mathcal{K}_{i}\right)$ to the homology classes of $H_{0}\left(\mathcal{K}_{i+1}\right)$. Given this, we formulate the problem of tracking as computing the sequence of maps $T_{i}$ defined in Equation 4 where $i=1 \ldots(n-1)$. For $i<j$, two path-connected components corresponding to a homology class in $H_{0}\left(\mathcal{K}_{i}\right)$ and a homology class in $H_{0}\left(\mathcal{K}_{j}\right)$ are determined to be the same object if and only if there exists a composition of maps $T_{i} \circ \cdots \circ T_{j-1}$ between the homology classes in question.

$$
H_{0}\left(\mathcal{K}_{1}\right) \stackrel{T_{1}}{\longrightarrow} H_{0}\left(\mathcal{K}_{2}\right) \stackrel{T_{2}}{\longrightarrow} H_{0}\left(\mathcal{K}_{2}\right) \stackrel{T_{3}}{\longrightarrow} \ldots \stackrel{T_{n-1}}{\longrightarrow} H_{0}\left(\mathcal{K}_{n}\right)
$$

\subsection{Pullback of the Zig-Zag Diagram}

Before computing the maps $T_{i}$ we first define the pullback which is a subspace of the direct sum of 0-dimensional homology groups in question. Toward this goal we first construct the zig-zag diagram of simplicial complexes with inclusion maps defined in Equation 5 [1]. Note that, $\mathcal{K}_{i}$ and $\mathcal{K}_{j}$ are subcomplexes of $\mathcal{K}_{i} \cup \mathcal{K}_{j}$. As will be illustrated in the next section, the mapping of consecutive simplicial complexes to their union in the zig-zag diagram facilitates the definition of the required map between homology classes. By applying the 0-dimensional homology functor to the zig-zag diagram of Equation 5 this gives the zig-zag diagram of homology groups with induced linear maps defined in Equation 6 [1].

$$
\begin{gathered}
\mathcal{K}_{1} \rightarrow \mathcal{K}_{1} \cup \mathcal{K}_{2} \leftarrow \mathcal{K}_{2} \rightarrow \cdots \leftarrow \mathcal{K}_{n} \\
H_{0}\left(\mathcal{K}_{1}\right) \rightarrow H_{0}\left(\mathcal{K}_{1} \cup \mathcal{K}_{2}\right) \leftarrow H_{0}\left(\mathcal{K}_{2}\right) \rightarrow \cdots \leftarrow H_{0}\left(\mathcal{K}_{n}\right)
\end{gathered}
$$


Let $\mathcal{K}_{i}$ and $\mathcal{K}_{j}$ be a pair of consecutive simplicial complexes in the sequence of simplicial complexes; that is, $j=i+1$. Furthermore, let $f$ and $g$ be the inclusion maps defined in Equation 7, and let $f_{*}$ and $g_{*}$ be induced maps defined in Equation 8. Recall that 0-dimensional homology classes correspond to path-connected components. Both $f_{*}$ and $g_{*}$ are surjective maps which map a 0-dimensional homology class in the domain to a 0-dimensional homology class in the codomain if and only if the path-connected component corresponding to the former is a subset of the path-connected component corresponding to the latter. This property is a consequence of the fact that individual 0-dimensional homology classes correspond to path-connected components.

To illustrate the maps $f_{*}$ and $g_{*}$ consider the example in Figure 1 . Here simplicial complexes corresponding to $\mathcal{K}_{i}$ and $\mathcal{K}_{j}$ are represented in (a) and (b) respectively and their union $\mathcal{K}_{i} \cup \mathcal{K}_{j}$ is represented in (c). In the figure individual path-connected components are labelled with a unique letter in the range $a-g$. In this example $f$ maps the 0 -dimensional homology classes corresponding to the path-connected components $a$ and $b$ in $\mathcal{K}_{i}$ to the 0 -dimensional homology classes corresponding to the path-connected components $f$ and $g$ in $\mathcal{K}_{i} \cup \mathcal{K}_{j}$ respectively. Similarly, $g$ maps the 0 -dimensional homology classes corresponding to the path-connected components $c$ and $d$ in $\mathcal{K}_{j}$ to the 0 -dimensional homology class corresponding to the path-connected component $f$ in $\mathcal{K}_{i} \cup \mathcal{K}_{j}$. It also maps the 0dimensional homology class corresponding to the path-connected component $e$ in $\mathcal{K}_{j}$ to the 0 -dimensional homology class corresponding to the path-connected component $g$ in $\mathcal{K}_{i} \cup \mathcal{K}_{j}$.

$$
\begin{gathered}
\mathcal{K}_{i} \stackrel{f}{\rightarrow} \mathcal{K}_{i} \cup \mathcal{K}_{j} \stackrel{g}{\longleftarrow} \mathcal{K}_{j} \\
H_{0}\left(\mathcal{K}_{i}\right) \stackrel{f_{*}}{\longrightarrow} H_{0}\left(\mathcal{K}_{i} \cup \mathcal{K}_{j}\right) \stackrel{g_{*}}{\longleftarrow} H_{0}\left(\mathcal{K}_{j}\right)
\end{gathered}
$$

The pullback $P$ of the maps $f$ and $g$ is defined in Equation 9 and is a subspace of the direct $\operatorname{sum} H_{0}\left(\mathcal{K}_{i}\right) \oplus H_{0}\left(\mathcal{K}_{j}\right)$. Specifically, the pullback is the set of all pairs of 0-dimensional homology classes of $\mathcal{K}_{i}$ and $\mathcal{K}_{j}$ which map to the same 0 -dimensional homology class of $\mathcal{K}_{i} \cup \mathcal{K}_{j}$. To illustrate the pullback consider again the example provided in Figure 1. Here the pullback is $\{(a, c),(a, d),(b, e)\}$ where 0 -dimensional homology classes are stated in terms of the labels of the path-connected components they correspond to.

$$
P=\left\{(a, b) \in H_{0}\left(\mathcal{K}_{i}\right) \oplus H_{0}\left(\mathcal{K}_{j}\right): f_{*}(a)=g_{*}(b)\right\}
$$

\subsection{Map of Homology Groups}

Each element in the pullback $P$ defines a map from an element of $H_{0}\left(\mathcal{K}_{i}\right)$ to an element of $H_{0}\left(\mathcal{K}_{j}\right)$. That is, if $(a, b) \in P$ this defines a map from $a \in H_{0}\left(\mathcal{K}_{i}\right)$ to $b \in H_{0}\left(\mathcal{K}_{j}\right)$. Recalling that $j=i+1$, if we were to construct the required map $T_{i}$ to be union of all elements in the pullback, this might not be an injective map ${ }^{1}$. For example, if a path-connected component splits into two path-connected components the pullback will contain two elements and the resulting map will not satisfy the property that the image of every element of the map's domain is at most one element of its codomain. Persistent homology computes an injective map between homology classes [3].

\footnotetext{
${ }^{1} \mathrm{~A}$ map is injective if and only if every element of the map's codomain is the image of at most one element of its domain and furthermore the image of every element of the map's domain is at most one element of its codomain.
}

To maintain consistency with persistent homology, we construct the required map $T_{i}$ to be injective by assigning it to be the union of a subset of the maps defined by the elements in the pullback. This subset is constructed using that outlined in Algorithm 1 which employs a heuristic giving preference with respect to persistence to homology classes which firstly have persisted for longer and secondly correspond to larger path-connected components.

Algorithm 1 first defines independent total orders on the elements of $H_{0}\left(\mathcal{K}_{i}\right)$ and $H_{0}\left(\mathcal{K}_{j}\right)$ (lines 3 and 4$)$. A total order on the elements of $H_{0}\left(\mathcal{K}_{i}\right)$ is defined using the lexicographical order over the time since appearing followed by the size of the corresponding path-connected component. A homology class appears at $\mathcal{K}_{b}$ if its preimage under the map $T_{b-1}$ is the empty set. A total order on the elements of $H_{0}\left(\mathcal{K}_{j}\right)$ is defined using the order over size of the corresponding path-connected components. To illustrate these independent orderings consider again the example of Figure 1. Assume the homology class corresponding to the path-connected component $a$ was born before that corresponding to the path-connected component $b$. In this case, the total order on the homology classes of $\mathcal{K}_{i}$ will be that corresponding to $b$ less than or equal to that corresponding to $a$. As a consequence of the fact that all the pathconnected components in $\mathcal{K}_{j}$ are of equal size, any ordering of the homology classes in question is valid.

Given the total orders defined above, a total order on the elements of the pullback $P$ is defined using the lexicographical order (line 5). These elements are then iterated through in decreasing order from largest to smallest (line 6) and each element is added to the map $T_{i}$ if its addition does not invalidate the injective property (lines 7 and 8).

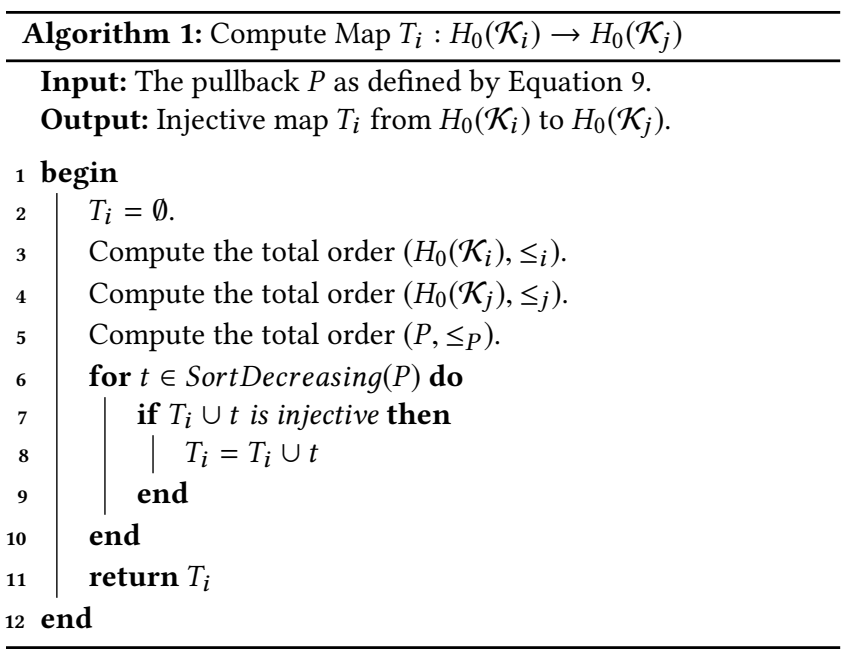

To illustrate the construction of $T_{i}$ consider again the example provided in Figure 1 where the pullback is $\{(a, c),(a, d),(b, e)\}$. Here the lexicographical order is $(b, e) \leq(a, d) \leq(a, c)$ and as a consequence the subset used to construct $T_{i}$ is $\{(a, c),(b, e)\}$.

\subsection{Tracking}

As stated in the model formulation of section 2.1, for $i<j$ homology classes in $H_{0}\left(\mathcal{K}_{i}\right)$ and $H_{0}\left(\mathcal{K}_{j}\right)$ are determined to be the same object if 


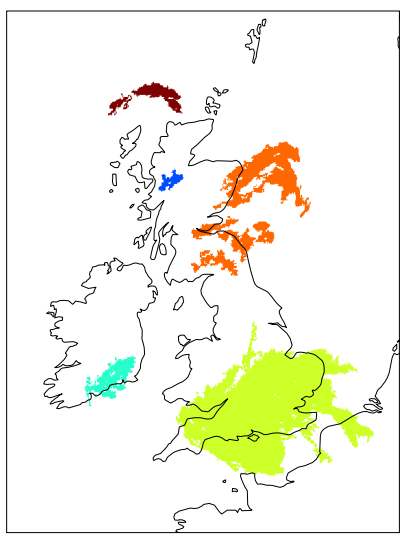

(a)

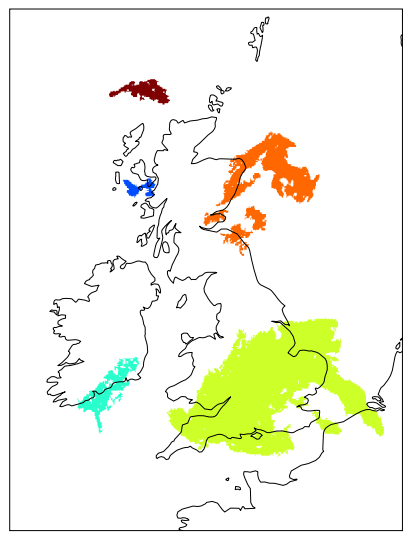

(b)

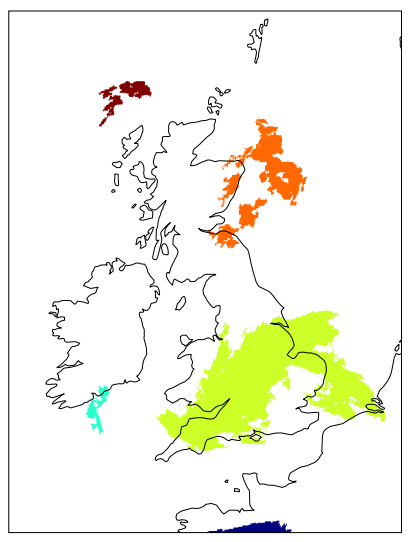

(c)

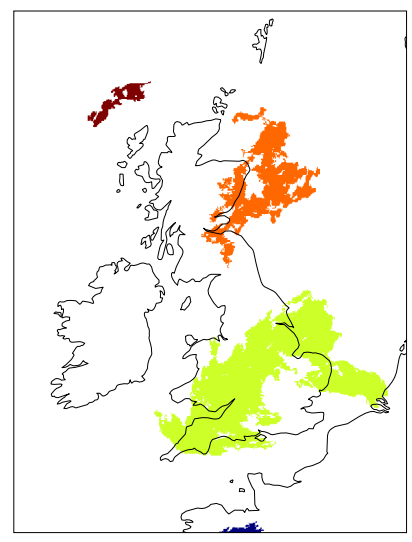

(d)

Figure 2: The robust tracking of objects in a sequence of four rain radar images is illustrated. The objects in question correspond to spatially close path-connected components and are uniquely coloured.

and only if there exists a compositions of maps $T_{i} \circ \cdots \circ T_{j-1}$ between the homology classes in question. Given this, we compute the set of homology classes corresponding to each object in the sequence $\mathcal{K}_{1} \ldots \mathcal{K}_{n}$ using an algorithm which is now briefly outlined.

The algorithm first constructs a graph $G=(V, E)$ where the set of vertices $V$ correspond to homology classes and the set of edges $E$ correspond to the existence of a map $T_{i}$ between the homology classes in question. Each connected component in this graph corresponds to an individual object and the set of homology classes contained in a given connected component equals those corresponding to the object in question. The connected components in $G$ are computed using a breadth first search and the set of homology classes in each of these components is constructed.

\subsection{Tracking in a Metric Space}

In many instances of the tracking problem, the objects in question lie in an ambient metric space where an individual object might not correspond to a single 0 -dimensional homology class but instead a set of such classes which are spatially close. For example, the problem of tracking a rainstorm may be formulated in terms of tracking a set of homology classes in $\mathbb{R}^{2}$ which are spatially close and where each corresponds to an individual rain cloud. We propose a generalisation of the proposed tracking method capable of tracking such objects corresponding to spatially close homology classes. In the original model we assume a sequence of $n$ simplicial complexes $\mathcal{K}_{1} \ldots \mathcal{K}_{n}$ obtained by triangulating path-connected components at a sequence of $n$ discrete time steps. In the generalised model we assume an additional sequence of $n$ simplicial complexes $\mathcal{K}_{1}^{\prime} \ldots \mathcal{K}_{n}^{\prime}$ obtained by triangulating an enlargement of the original path-connected components at the same sequence of discrete times. The relationship between these two sequences of simplicial complexes is that spatially close path-connected components in a given $\mathcal{K}_{i}$ will form a single larger path-connected component in $\mathcal{K}_{i}^{\prime}$.

In the generalised model, we apply the original tracking model to the sequence of simplicial complexes $\mathcal{K}_{1}^{\prime} \ldots \mathcal{K}_{n}^{\prime}$. Recall that, this model will compute the set of homology classes corresponding to each object in the sequence $\mathcal{K}_{1}^{\prime} \ldots \mathcal{K}_{n}^{\prime}$. We next propagate the result of this tracking to the sequence $\mathcal{K}_{1} \ldots \mathcal{K}_{n}$.

\section{RESULTS}

This section describes the application of the generalised tracking model, described in section 2.5, to rainfall radar images obtained from the UK Meteorological (Met) Office. The Met office provides this data at 15 minute intervals. For a given time, the image data in question categorises the rainfall level at each location in a 500x500 regular grid over Ireland and the UK. Given this data, we consider the problem of tracking objects corresponding to spatially close path-connected components of $\mathbb{R}^{2}$ with a rainfall level greater than a threshold. Recall that, the generalised tracking model requires that at each time we triangulate both the original and enlarged pathconnected components. The enlarged path-connected components are constructed by performing a binary morphology dilation of the grid of points with a rainfall level greater than the threshold.

Figure 2 illustrates the application of the model to a short sequence of rainfall radar images. In this figure all path-connected components corresponding to the same object are uniquely coloured. It is evident from this figure that the model accurately tracks objects corresponding to spatially close path-connected components. For example, the path-connected components in the center of the radar images are spatially close and are correctly determined to correspond to a single object which is coloured orange. A video clip displaying a longer tracking sequence can be viewed online at the following URL - https://youtu.be/w_IZSZEzAsg.

\section{REFERENCES}

[1] Gunnar Carlsson and Vin De Silva. 2010. Zigzag persistence. Foundations of computational mathematics 10, 4 (2010), 367-405.

[2] Padraig Corcoran and Christopher B Jones. 2016. Spatio-temporal modeling of the topology of swarm behavior with persistence landscapes. In ACM SIGSPATIAL International Conference on Advances in Geographic Information Systems. 65.

[3] Padraig Corcoran and Christopher B Jones. 2017. Modelling topological features of swarm behaviour in space and time with persistence landscapes. IEEE Access 5 (2017), 18534-18544.

[4] Afra Zomorodian and Gunnar Carlsson. 2008. Localized homology. Computational Geometry 41, 3 (2008), 126-148. 